\title{
Some New Congruences for Andrews' Singular Overpartition Pairs
}

\author{
M. S. Mahadeva Naika ${ }^{1} \cdot$ S. Shivaprasada Nayaka ${ }^{1}$
}

Received: 26 December 2016 / Accepted: 5 September 2017 / Published online: 24 January 2018

(C) Vietnam Academy of Science and Technology (VAST) and Springer Nature Singapore Pte Ltd. 2018

\begin{abstract}
In a recent work, Andrews defined the combinatorial objects called singular overpartitions denoted by $\bar{C}_{k, i}(n)$, which count the number of overpartitions of $n$ in which no part is divisible by $k$ and only parts congruent to $\pm i$ modulo $k$ may be overlined. Many authors have found congruences and infinite families of congruences modulo powers of 2 and 3 . In this paper, we find some new infinite families of congruences for $\bar{C}_{1,2}^{6}(n)$ modulo
\end{abstract} 27 and congruences modulo 4 for $\bar{C}_{1,5}^{12}(n), \bar{C}_{3,3}^{9}(n)$ and $\bar{C}_{5,5}^{15}(n)$.

Keywords Congruences · Dissections · Singular overpartition pairs · Theta function

Mathematics Subject Classification (2010) $11 \mathrm{P} 83 \cdot 05 \mathrm{~A} 15 \cdot 05 \mathrm{~A} 17$

\section{Introduction}

A partition of a positive integer $n$ is a non-increasing sequence of positive integers whose sum is $n$. An overpartition, introduced by Corteel and Lovejoy [9], of a non-negative integer $n$ is a non-increasing sequence of natural numbers whose sum is $n$ in which the first occurrence of a number may be overlined. For example, the eight overpartitions of 3 are

$$
3, \quad \overline{3}, \quad 2+1, \quad \overline{2}+1, \quad 2+\overline{1}, \quad \overline{2}+\overline{1}, \quad 1+1+1, \quad \overline{1}+1+1 .
$$

M. S. Mahadeva Naika msmnaika@rediffmail.com

S. Shivaprasada Nayaka shivprasadnayaks@gmail.com

1 Department of Mathematics, Bangalore University, Central College Campus, Bangalore, 560 001, Karnataka, India 
Andrews [3] introduced singular overpartitions. To introduce singular overpartitions, first he defined some properties of the entries in a Frobenius symbol for $n$, which is of the form

$$
\left(\begin{array}{llll}
a_{1} & a_{2} & \ldots & a_{r} \\
b_{1} & b_{2} & \ldots & b_{r}
\end{array}\right)
$$

where $\sum\left(a_{i}+b_{i}+1\right)=n$ and $a_{1}>a_{2}>\cdots>a_{r} \geq 0, b_{1}>b_{2}>\cdots>b_{r} \geq 0$. There is a natural mapping that reveals a one-to-one correspondence between the Frobenius symbols for $n$ and the ordinary partitions of $n$. "Singular overpartitions" are Frobenius symbols for $n$ with at most one overlined entry in each row. More precisely, for two positive integers $k$ and $i$, a column $\left(\begin{array}{l}a_{j} \\ b_{j}\end{array}\right)$ in a Frobenius symbol is $(k, i)$-positive if $a_{j}-b_{j} \geq k-i-1$ and ( $k, i)$-negative if $a_{j}-b_{j} \leq-i+1$. If $-i+1<a_{j}-b_{j}<k-i+1$, then we say the column is $(k, i)$-neutral. Two columns have the same parity if they are both $(k, i)$-positive or $(k, i)$ negative. We can divide the Frobenius symbol into $(k, i)$-blocks such that all the entries in each block have either the same $(k, i)$-parity or $(k, i)$-neutral. The first non-neutral column in each parity block is called the anchor of the block. A $(k, i)$-parity block is neutral if all columns in it are neutral and a $(k, i)$-parity block is positive (resp. negative) if it contains no $(k, i)$-negative (resp. positive) columns.

A Frobenius symbol is $(k, i)$-singular if

1) there are no overlined entries, or

2) the one overlined entry on the top row occurs in an anchor of a $(k, i)$-positive block, or

3 ) the one overlined entry on the bottom row occurs in an anchor of a $(k, i)$-negative block, and

4) if there is one overlined entry in each row, then they occur in adjacent $(k, i)$-parity blocks.

Let $\bar{Q}_{k, i}(n)$ denote the number of such singular overpartitions for $1 \leq i \leq\left\lfloor\frac{k}{2}\right\rfloor$. Andrews proved that $\bar{Q}_{k, i}(n)=\bar{C}_{k, i}(n)$, where $\bar{C}_{k, i}(n)$ counts the number of overpartitions of $n$ in which no part is divisible by $k$ and only parts congruent to $\pm i$ modulo $k$ may be overlined. Therefore for $k \geq 3$ and $1 \leq i \leq\left\lfloor\frac{k}{2}\right\rfloor$, the generating function for $\bar{C}_{k, i}(n)$ is given by

$$
\sum_{n=0}^{\infty} \bar{Q}_{k, i}(n) q^{n}=\sum_{n=0}^{\infty} \bar{C}_{k, i}(n) q^{n}=\frac{\left(q^{k} ; q^{k}\right)_{\infty}\left(-q^{i} ; q^{k}\right)_{\infty}\left(-q^{k-i} ; q^{k}\right)_{\infty}}{(q ; q)_{\infty}} .
$$

The ten singular overpartitions counted by $\bar{C}_{3,1}(4)$ are

$$
4, \overline{4}, 2+2, \overline{2}+2,2+1+1, \overline{2}+1+1,2+\overline{1}+1, \overline{2}+\overline{1}+1,1+1+1+1, \overline{1}+1+1+1 \text {. }
$$

Throughout the paper, we use the standard $q$-series notation, and $f_{k}$ is defined as

$$
f_{k}:=\left(q^{k} ; q^{k}\right)_{\infty}=\lim _{n \rightarrow \infty} \prod_{m=1}^{n}\left(1-q^{m k}\right) .
$$

For $|a b|<1$, Ramanujan's general theta function $f(a, b)$ is defined as

$$
f(a, b):=\sum_{n=-\infty}^{\infty} a^{n(n+1) / 2} b^{n(n-1) / 2}
$$

where the product representations arise from Jacobi's triple product identity [5, p. 35, Entry 19], [1]

$$
f(a, b)=(-a ; a b)_{\infty}(-b ; a b)_{\infty}(a b ; a b)_{\infty} .
$$


The special cases of $f(a, b)$ are

$$
\begin{aligned}
& \varphi(q):=f(q, q)=\sum_{n=-\infty}^{\infty} q^{n^{2}}=\left(-q ; q^{2}\right)_{\infty}^{2}\left(q^{2} ; q^{2}\right)_{\infty}=\frac{f_{2}^{5}}{f_{1}^{2} f_{4}^{2}}, \\
& \psi(q):=f\left(q, q^{3}\right)=\sum_{n=0}^{\infty} q^{n(n+1) / 2}=\frac{\left(q^{2} ; q^{2}\right)_{\infty}}{\left(q ; q^{2}\right)_{\infty}}=\frac{f_{2}^{2}}{f_{1}},
\end{aligned}
$$

and

$$
f(-q):=f\left(-q,-q^{2}\right)=\sum_{n=-\infty}^{\infty}(-1)^{n} q^{n(3 n-1) / 2}=(q ; q)_{\infty}=f_{1},
$$

Andrews [3] proves that, for all $n \geq 0, \bar{C}_{3,1}(n)=\bar{A}_{3}(n)$, where $\bar{A}_{3}(n)$ is the number of overpartitions of $n$ into parts not divisible by 3 . The function $\bar{A}_{\ell}(n)$, which counts the number of overpartitions of $n$ into parts not divisible by $\ell$, plays a key role in the work of Lovejoy [13].

In his beautiful paper, Andrews also found the following congruences:

$$
\bar{C}_{3,1}(9 n+3) \equiv \bar{C}_{3,1}(9 n+6) \equiv 0 \quad(\bmod 3) .
$$

Chen et al. [8] have generalized (4) and proved some congruences modulo 2, 3, 4, and 8 for $\bar{C}_{3,1}(n)$. They also proved some congruence for $\bar{C}_{4,1}(n), \bar{C}_{6,1}(n)$ and $\bar{C}_{6,2}(n)$ modulo powers of 2 and 3. More recently Ahmed and Baruah [2] have found some new congruences for $\bar{C}_{3,1}(n), \bar{C}_{8,2}(n), \bar{C}_{12,4}(n), \bar{C}_{24,8}(n)$ and $\bar{C}_{48,16}(n)$ modulo 18, 36. Chen [7] has also found some congruences modulo powers of 2 for $\bar{C}_{3,1}(n), \bar{C}_{4,1}(n)$. Yao [22] has proved congruences modulo 16, 32, 64 for $\bar{C}_{3,1}(n)$. Naika and Gireesh [14] have found some congruences modulo $6,12,16,18,24,48$, and 72 for $\bar{C}_{3,1}(n)$. Naika and Nayaka [16] have proved some congruences for $\overline{C O}_{3,1}(n)$ modulo powers of 2 and 3 . They have also proved in the paper [17] modulo 4 for $\bar{C}_{4,1}^{3}(n)$ and $\bar{C}_{4,1}^{5}(n)$.

Mahadeva Naika and Shivashankar [18] have defined the Andrews' singular overpatition pairs of $n$. Let $\bar{C}_{i, j}^{\delta}(n)$ denote the number of Andrews' singular overpatition pairs of $n$ in which no part is divisible by $\delta$ and only parts congruent to $\pm i, \pm j$ modulo $\delta$ may be overlined. An Andrews' singular overpatition pair $\pi$ of $n$ is a pair of Andrews' singular overpatitions $(\lambda, \mu)$ such that the sum of all of the parts is $n$. For $\delta \geq 3$ and $1 \leq i, j \leq\left\lfloor\frac{\delta}{2}\right\rfloor$, the generating function for $\bar{C}_{i, j}^{\delta}(n)$ is

$$
\sum_{n=0}^{\infty} \bar{C}_{i, j}^{\delta}(n) q^{n}=\frac{f\left(q^{i}, q^{\delta-i}\right) f\left(q^{j}, q^{\delta-j}\right)}{(q ; q)_{\infty}^{2}}
$$

In this paper, we find some new infinite families of congruences for $\bar{C}_{1,2}^{6}(n)$ modulo 27 and congruences modulo 4 for $\bar{C}_{1,5}^{12}(n), \bar{C}_{3,3}^{9}(n)$ and $\bar{C}_{5,5}^{15}(n)$. The main results of this paper can be stated as follows.

Theorem 1 For any $\alpha \geq 0$ and $n \geq 0$,

$$
\bar{C}_{1,2}^{6}\left(4^{\alpha+2} n+\frac{4^{\alpha+2}-1}{3}\right) \equiv 7^{\alpha+1} \cdot \bar{C}_{1,2}^{6}(4 n+1) \quad(\bmod 27) .
$$


Theorem 2 For all $\alpha \geq 0$ and $n \geq 0$,

$$
\begin{aligned}
\sum_{n=0}^{\infty} \bar{C}_{1,5}^{12}(3 n) q^{n} & \equiv \frac{f_{1} f_{3} f_{6}}{f_{2}} \quad(\bmod 4), \\
\sum_{n=0}^{\infty} \bar{C}_{1,5}^{12}(3 n+1) q^{n} & \equiv 3 \frac{f_{1}^{2} f_{6}^{4}}{f_{2}^{2} f_{3}^{2}} \quad(\bmod 4), \\
\sum_{n=0}^{\infty} \bar{C}_{1,5}^{12}(3 n+2) q^{n} & \equiv 3 \frac{f_{1} f_{3} f_{12}^{3}}{f_{4} f_{6}^{2}} \quad(\bmod 4), \\
\bar{C}_{1,5}^{12}\left(3^{\alpha+1} n+3^{\alpha+1}-1\right) & \equiv 3^{\alpha+1} \cdot \bar{C}_{1,5}^{12}(n) \quad(\bmod 4) .
\end{aligned}
$$

Theorem 3 For all integers $\alpha \geq 0$ and $n \geq 0$,

$$
\begin{aligned}
\bar{C}_{1,5}^{12}(12 n+6) & \equiv 0 & (\bmod 4), \\
\bar{C}_{1,5}^{12}(48 n+27) & \equiv 0 & (\bmod 4), \\
\bar{C}_{1,5}^{12}(96 n+87) & \equiv 0 & (\bmod 4), \\
\bar{C}_{1,5}^{12}\left(3 \cdot 4^{\alpha+3} n+7 \cdot 4^{\alpha+2}-1\right) & \equiv 0 & (\bmod 4) .
\end{aligned}
$$

Theorem 4 For all $\alpha \geq 0$ and $n \geq 0$,

$$
\begin{aligned}
\bar{C}_{1,5}^{12}(24 n+13) & \equiv 0 \quad(\bmod 4), \\
\bar{C}_{1,5}^{12}(24 n+3) & \equiv \bar{C}_{1,5}^{12}(12 n+1) \quad(\bmod 4), \\
\bar{C}_{1,5}^{12}(24 n+15) & \equiv \bar{C}_{1,5}^{12}(12 n+7) \quad(\bmod 4) .
\end{aligned}
$$

Theorem 5 For each $n \geq 0$ and $\alpha \geq 0$,

$$
\begin{aligned}
\bar{C}_{1,5}^{12}\left(12 \cdot 25^{\alpha+1} n+25^{\alpha+1}-1\right) & \equiv \bar{C}_{1,5}^{12}(12 n) \quad(\bmod 4), \\
\bar{C}_{1,5}^{12}(60(5 n+i)+24) & \equiv 0 \quad(\bmod 4),
\end{aligned}
$$

where $i=1,2,3,4$.

Theorem 6 Let $p$ be a prime $\geq 5,\left(\frac{-4}{p}\right)=-1$. Then for all integers $\alpha \geq 1$, and $n \geq 0$,

$$
\sum_{n=0}^{\infty} \bar{C}_{1,5}^{12}\left(192 p^{2 \alpha} n+39 p^{2 \alpha}-1\right) q^{n} \equiv 2 f_{1} f_{4} \quad(\bmod 4)
$$

Theorem 7 Let $p$ be a prime $\geq 5,\left(\frac{-4}{p}\right)=-1$. Then for all integers $\alpha \geq 0$, and $n \geq 0$,

$$
\bar{C}_{1,5}^{12}\left(192 p^{2 \alpha+2} n+192 p^{2 \alpha+1} i+40 p^{2 \alpha+2}-1\right) \equiv 0 \quad(\bmod 4),
$$

where $i$ is an integer and $1 \leq i \leq p-1$. 
Theorem 8 Let $p$ be a prime $\geq 5,\left(\frac{-6}{p}\right)=-1$. Then for all integers $\alpha \geq 1$, and $n \geq 0$,

$$
\sum_{n=0}^{\infty} \bar{C}_{3,3}^{9}\left(4 p^{2 \alpha} n+\frac{7 p^{2 \alpha}-1}{6}\right) q^{n} \equiv 2 f_{1} f_{6} \quad(\bmod 4)
$$

Theorem 9 Let $p$ be a prime $\geq 5,\left(\frac{-6}{p}\right)=-1$. Then for all integers $\alpha \geq 0$, and $n \geq 0$,

$$
\bar{C}_{3,3}^{9}\left(4 p^{2 \alpha+2} n+4 p^{2 \alpha+1} i+\frac{7 p^{2 \alpha+2}-1}{6}\right) \equiv 0 \quad(\bmod 4)
$$

where $i$ is an integer and $1 \leq i \leq p-1$.

Theorem 10 For each $n \geq 0$ and $\alpha \geq 0$,

$$
\begin{aligned}
\bar{C}_{5,5}^{15}(16 n+9) & \equiv 0 \quad(\bmod 4), \\
\bar{C}_{5,5}^{15}(20 n+3) & \equiv \bar{C}_{5,5}^{15}(8 n+1) \quad(\bmod 4), \\
\bar{C}_{5,5}^{15}(10 n+3) & \equiv \bar{C}_{5,5}^{15}(4 n+1) \quad(\bmod 4), \\
\bar{C}_{5,5}^{15}\left(2 \cdot 4^{\alpha+1} n+\frac{4^{\alpha+2}-1}{3}\right) & \equiv \bar{C}_{5,5}^{15}(2 n+1) \quad(\bmod 4), \\
\bar{C}_{5,5}^{15}\left(4 \cdot 5^{5 \alpha+6}(5 n+i)+\frac{2 \cdot 5^{5 \alpha+6}-1}{3}\right) & \equiv 0 \quad(\bmod 4),
\end{aligned}
$$

where $i=1,2,3,4$.

$$
\bar{C}_{5,5}^{15}\left(2 \cdot 5^{2 \alpha+4}(5 n+j)+\frac{4 \cdot 5^{2 \alpha+4}-1}{3}\right) \equiv 0 \quad(\bmod 4),
$$

where $j=3,4$.

\section{Preliminary Results}

We need the following few dissection formulas to prove our main results,

Lemma 1 [19, p. 212] We have the following 5-dissection

$$
f_{1}=f_{25}\left(a\left(q^{5}\right)-q-\frac{q^{2}}{a\left(q^{5}\right)}\right)
$$

where

$$
a(q):=\frac{\left(q^{2}, q^{3} ; q^{5}\right)_{\infty}}{\left(q, q^{4} ; q^{5}\right)_{\infty}}
$$


Lemma 2 The following 2-dissections hold:

$$
\begin{aligned}
& \frac{f_{3}^{3}}{f_{1}}=\frac{f_{4}^{3} f_{6}^{2}}{f_{2}^{2} f_{12}}+q \frac{f_{12}^{3}}{f_{4}}, \\
& \frac{f_{3}}{f_{1}^{3}}=\frac{f_{4}^{6} f_{6}^{3}}{f_{2}^{9} f_{12}^{2}}+3 q \frac{f_{4}^{2} f_{6} f_{12}^{2}}{f_{2}^{7}}, \\
& \frac{f_{1}}{f_{3}^{3}}=\frac{f_{2} f_{4}^{2} f_{12}^{2}}{f_{6}^{7}}-q \frac{f_{2}^{3} f_{12}^{6}}{f_{4}^{2} f_{6}^{9}}, \\
& \frac{f_{1}^{3}}{f_{3}}=\frac{f_{4}^{3}}{f_{12}}-3 q \frac{f_{2}^{2} f_{12}^{3}}{f_{4} f_{6}^{2}} .
\end{aligned}
$$

Hirschhorn et al. [11] have proved (30). For the proof of (31), see [4]. The proofs of (32) and (33) follow by changing $q$ to $-q$ in (30) and (31) respectively with $(-q ;-q)_{\infty}=\frac{f_{2}^{3}}{f_{1} f_{4}}$.

Lemma 3 The following 2-dissection formulas hold:

$$
\begin{aligned}
& \frac{1}{f_{1} f_{3}}=\frac{f_{8}^{2} f_{12}^{5}}{f_{2}^{2} f_{4} f_{6}^{4} f_{24}^{2}}+q \frac{f_{4}^{5} f_{24}^{2}}{f_{2}^{4} f_{6}^{2} f_{8}^{2} f_{12}}, \\
& f_{1} f_{3}=\frac{f_{2} f_{8}^{2} f_{12}^{4}}{f_{4}^{2} f_{6} f_{24}^{2}}-q \frac{f_{4}^{4} f_{6} f_{24}^{2}}{f_{2} f_{8}^{2} f_{12}^{2}} .
\end{aligned}
$$

Equation (34) was proved by Baruah and Ojah [6]. Replacing $q$ by $-q$ in (34) and using the fact that $(-q ;-q)_{\infty}=\frac{f_{2}^{3}}{f_{1} f_{4}}$, we get (35).

Lemma 4 The following 2-dissection formulas hold:

$$
\begin{aligned}
& \frac{f_{3}^{2}}{f_{1}^{2}}=\frac{f_{4}^{4} f_{6} f_{12}^{2}}{f_{2}^{5} f_{8} f_{24}}+2 q \frac{f_{4} f_{6}^{2} f_{8} f_{24}}{f_{2}^{4} f_{12}}, \\
& \frac{f_{1}^{2}}{f_{3}^{2}}=\frac{f_{2} f_{4}^{2} f_{12}^{4}}{f_{6}^{5} f_{8} f_{24}}-2 q \frac{f_{2}^{2} f_{8} f_{12} f_{24}}{f_{4} f_{6}^{4}} .
\end{aligned}
$$

Xia and Yao [21] proved (36) by employing an addition formula for theta functions. Replacing $q$ by $-q$ in (36) and using the fact that $(-q ;-q)_{\infty}=\frac{f_{2}^{3}}{f_{1} f_{4}}$, we get (37).

Lemma 5 The following 3-dissection holds:

$$
\frac{f_{4}}{f_{1}}=\frac{f_{12} f_{18}^{4}}{f_{3}^{3} f_{36}^{2}}+q \frac{f_{6}^{2} f_{9}^{3} f_{36}}{f_{3}^{4} f_{18}^{2}}+2 q^{2} \frac{f_{6} f_{18} f_{36}}{f_{3}^{3}} .
$$

Equation (38) is nothing but Lemma 2.6 in [6].

Lemma 6 [20] We have the following 3-dissection

$$
\frac{f_{1}}{f_{4}}=\frac{f_{6} f_{9} f_{18}}{f_{12}^{3}}-q \frac{f_{3} f_{18}^{4}}{f_{12}^{3} f_{9}^{2}}-q^{2} \frac{f_{6}^{2} f_{9} f_{36}^{3}}{f_{12}^{4} f_{18}^{2}} .
$$


Proof From (3), we have

$$
\begin{aligned}
\frac{f_{1}}{f_{4}}=\frac{(q ; q)_{\infty}}{\left(q^{4} ; q^{4}\right)_{\infty}} & =\frac{\left(q, q^{2}, q^{3}, q^{4}, q^{5}, q^{6} ; q^{6}\right)_{\infty}}{\left(q^{4} ; q^{4}\right)_{\infty}} \\
& =\frac{\left(q, q^{5}, q^{6} ; q^{6}\right)_{\infty}\left(q^{2}, q^{4}, q^{8}, q^{10} ; q^{12}\right)_{\infty}\left(q^{3} ; q^{6}\right)_{\infty}\left(q^{6} ; q^{6}\right)_{\infty}}{\left(q^{4}, q^{8}, q^{12} ; q^{12}\right)_{\infty}\left(q^{6} ; q^{6}\right)_{\infty}} \\
& =\frac{\left(q, q^{5}, q^{6} ; q^{6}\right)_{\infty}\left(q^{2}, q^{10}, q^{12} ; q^{12}\right)_{\infty}\left(q^{3} ; q^{3}\right)_{\infty}}{\left(q^{6} ; q^{6}\right)_{\infty}\left(q^{12} ; q^{12}\right)_{\infty}^{2}},
\end{aligned}
$$

where $\left(a_{1}, a_{2}, \ldots, a_{k} ; q\right)_{\infty}=\left(a_{1} ; q\right)_{\infty}\left(a_{2} ; q\right)_{\infty} \ldots\left(a_{k} ; q\right)_{\infty}$.

Now by Jacobi triple product identity

$$
\begin{aligned}
& \left(q, q^{5}, q^{6} ; q^{6}\right)_{\infty}\left(q^{2}, q^{10}, q^{12} ; q^{12}\right)_{\infty} \\
& =\sum_{m=-\infty}^{\infty}(-1)^{m} q^{3 m^{2}+2 m} \cdot \sum_{n=-\infty}^{\infty}(-1)^{n} q^{6 n^{2}+4 n} \\
& =\sum_{m, n=-\infty}^{\infty}(-1)^{m+n} q^{3 m^{2}+2 m+6 n^{2}+4 n} \\
& =\sum_{t, u=-\infty}^{\infty}(-1)^{2 t-u} q^{3(t-2 u)^{2}+2(t-2 u)+6(t+u-1)^{2}+4(t+u-1)} \\
& +\sum_{t, u=-\infty}^{\infty}(-1)^{2 t-u-1} q^{3(t-2 u-1)^{2}+2(t-2 u-1)+6(t+u)^{2}+4(t+u)} \\
& +\sum_{t, u=-\infty}^{\infty}(-1)^{2 t-u-1} q^{3(t-2 u)^{2}+2(t-2 u)+6(t+u-1)^{2}+4(t+u-1)} \\
& =\sum_{t, u=-\infty}^{\infty}(-1)^{u} q^{9 t^{2}+18 u^{2}+6 t}+q \sum_{t, u=-\infty}^{\infty}(-1)^{-u-1} q^{9 t^{2}+18 u^{2}+12 u} \\
& +q^{2} \sum_{t, u=-\infty}^{\infty}(-1)^{-u-1} q^{9 t^{2}-6 t+18 u^{2}-12 u} \\
& =\left(-q^{3},-q^{15}, q^{18} ; q^{18}\right)_{\infty}\left(q^{18}, q^{18}, q^{36} ; q^{36}\right)_{\infty} \\
& -q\left(-q^{9},-q^{9}, q^{18} ; q^{18}\right)_{\infty}\left(q^{6}, q^{30}, q^{36} ; q^{36}\right)_{\infty} \\
& -q^{2}\left(-q^{3},-q^{15}, q^{18} ; q^{18}\right)_{\infty}\left(q^{6}, q^{30}, q^{36} ; q^{36}\right)_{\infty} \\
& =\frac{\left(q^{6} ; q^{6}\right)_{\infty}\left(q^{9} ; q^{9}\right)_{\infty}\left(q^{36} ; q^{36}\right)_{\infty}}{\left(q^{3} ; q^{3}\right)_{\infty}\left(q^{12} ; q^{12}\right)_{\infty}\left(q^{18} ; q^{18}\right)_{\infty}} \cdot \frac{\left(q^{18} ; q^{18}\right)_{\infty}^{2}\left(q^{36} ; q^{36}\right)_{\infty}}{\left(q^{36} ; q^{36}\right)_{\infty}^{2}} \\
& -q \frac{\left(q^{6} ; q^{6}\right)_{\infty}\left(q^{36} ; q^{36}\right)_{\infty}^{2}}{\left(q^{12} ; q^{12}\right)_{\infty}\left(q^{18} ; q^{18}\right)_{\infty}} \cdot \frac{\left(q^{18} ; q^{18}\right)_{\infty}^{5}}{\left(q^{9} ; q^{9}\right)_{\infty}^{2}\left(q^{36} ; q^{36}\right)_{\infty}^{2}} \\
& -q^{2} \frac{\left(q^{6} ; q^{6}\right)_{\infty}^{2}\left(q^{9} ; q^{9}\right)_{\infty}\left(q^{36} ; q^{36}\right)_{\infty}}{\left(q^{3} ; q^{3}\right)_{\infty}\left(q^{12} ; q^{12}\right)_{\infty}\left(q^{18} ; q^{18}\right)_{\infty}} \cdot \frac{\left(q^{6} ; q^{6}\right)_{\infty}\left(q^{36} ; q^{36}\right)_{\infty}^{2}}{\left(q^{12} ; q^{12}\right)_{\infty}\left(q^{18} ; q^{18}\right)_{\infty}} .
\end{aligned}
$$

Substituting (41) into (40), we obtain (39). 
Lemma 7 The following 2-dissection holds:

$$
\frac{f_{5}}{f_{1}}=\frac{f_{8} f_{20}^{2}}{f_{2}^{2} f_{40}}+q \frac{f_{4}^{3} f_{10} f_{40}}{f_{2}^{3} f_{8} f_{20}} .
$$

Equation (42) was proved by Hirschhorn and Sellers [12].

Lemma 8 [15, Lemma 2.3] The following 2-dissection formulas hold:

$$
\begin{aligned}
& f_{1} f_{5}^{3}=2 q^{2} f_{4} f_{20}^{3}+f_{2}^{3} f_{10}-2 q^{3} \frac{f_{4}^{4} f_{40}^{2} f_{10}}{f_{2} f_{8}^{2}}-q \frac{f_{2}^{2} f_{10}^{2} f_{20}}{f_{4}}, \\
& f_{1}^{3} f_{5}=2 q^{2} \frac{f_{4}^{6} f_{40}^{2} f_{10}}{f_{2} f_{8}^{2} f_{20}^{2}}+\frac{f_{2}^{2} f_{4} f_{10}^{2}}{f_{20}}+2 q f_{4}^{3} f_{20}-5 q f_{2} f_{10}^{3} .
\end{aligned}
$$

Lemma 9 (Cui and Gu [10, Theorem 2.2]) For any prime $p \geq 5$,

$$
f_{1}=\sum_{\substack{k=\frac{1-p}{2} \\ k \neq \frac{ \pm p-1}{6}}}^{\frac{p-1}{2}}(-1)^{k} q^{\frac{3 k^{2}+k}{2}} f\left(-q^{\frac{3 p^{2}+(6 k+1) p}{2}},-q^{\frac{3 p^{2}-(6 k+1) p}{2}}\right)+(-1)^{\frac{ \pm p-1}{6}} q^{\frac{p^{2}-1}{24}} f_{p^{2}},
$$

where

$$
\frac{ \pm p-1}{6}:= \begin{cases}\frac{p-1}{6} & \text { if } p \equiv 1 \quad(\bmod 6) \\ \frac{-p-1}{6} & \text { if } p \equiv-1 \quad(\bmod 6)\end{cases}
$$

\section{Proof of Theorem 1}

Setting $i=1, j=2$ and $\delta=6$ in (5), we see that

$$
\sum_{n=0}^{\infty} \bar{C}_{1,2}^{6}(n) q^{n}=\frac{f\left(q, q^{5}\right) f\left(q^{2}, q^{4}\right)}{(q ; q)_{\infty}^{2}}
$$

By the definition of $f(a, b)$ and the well-known Jacobi triple product identity, we get

$$
\sum_{n=0}^{\infty} \bar{C}_{1,2}^{6}(n) q^{n}=\frac{f_{2} f_{3} f_{6}}{f_{1}^{3}}
$$

Substituting (31) into (45), we have

$$
\sum_{n=0}^{\infty} \bar{C}_{1,2}^{6}(n) q^{n}=f_{2} f_{6}\left(\frac{f_{4}^{6} f_{6}^{3}}{f_{2}^{9} f_{12}^{2}}+3 q \frac{f_{4}^{2} f_{6} f_{12}^{2}}{f_{2}^{7}}\right) .
$$

Equating odd parts of the above equation, we obtain

$$
\sum_{n=0}^{\infty} \bar{C}_{1,2}^{6}(2 n+1) q^{n}=3 \frac{f_{2}^{2} f_{3}^{2} f_{6}^{2}}{f_{1}^{6}}
$$

Employing (31) into (46), we arrive at

$$
\sum_{n=0}^{\infty} \bar{C}_{1,2}^{6}(2 n+1) q^{n}=3 \frac{f_{4}^{12} f_{6}^{8}}{f_{2}^{16} f_{12}^{4}}+18 q \frac{f_{4}^{8} f_{6}^{6}}{f_{2}^{14}}+27 q^{2} \frac{f_{4}^{4} f_{6}^{4} f_{12}^{4}}{f_{2}^{12}}
$$


Extracting the terms involving $q^{2 n}$ from both sides of (47), we have

$$
\sum_{n=0}^{\infty} \bar{C}_{1,2}^{6}(4 n+1) q^{n}=3 \frac{f_{2}^{12} f_{3}^{8}}{f_{1}^{16} f_{6}^{4}}+27 q \frac{f_{2}^{4} f_{3}^{4} f_{6}^{4}}{f_{1}^{12}},
$$

which implies that

$$
\sum_{n=0}^{\infty} \bar{C}_{1,2}^{6}(4 n+1) q^{n} \equiv 3 \frac{f_{2}^{12} f_{3}^{8}}{f_{1}^{16} f_{6}^{4}} \quad(\bmod 27) .
$$

By the binomial theorem, it is easy to see that for positive integers $k$ and $m$,

$$
f_{3 k}^{3 m} \equiv f_{k}^{9 m} \quad(\bmod 9) .
$$

Invoking (49) in (48), we see that

$$
\sum_{n=0}^{\infty} \bar{C}_{1,2}^{6}(4 n+1) q^{n} \equiv 3 \frac{f_{1}^{2} f_{2}^{3} f_{3}^{2}}{f_{6}} \quad(\bmod 27) .
$$

Substituting (35) into (50), we obtain

$$
\sum_{n=0}^{\infty} \bar{C}_{1,2}^{6}(4 n+1) q^{n} \equiv 3 \frac{f_{2}^{5} f_{8}^{4} f_{12}^{8}}{f_{4}^{4} f_{6}^{3} f_{24}^{4}}+3 q^{2} \frac{f_{2} f_{4}^{8} f_{6} f_{24}^{4}}{f_{8}^{4} f_{12}^{4}}-6 q \frac{f_{2}^{3} f_{4}^{2} f_{12}^{2}}{f_{6}} \quad(\bmod 27) .
$$

Extracting the terms involving $q^{2 n+1}$ from (51), dividing by $q$ and replacing $q^{2}$ by $q$, we get

$$
\sum_{n=0}^{\infty} \bar{C}_{1,2}^{6}(8 n+5) q^{n} \equiv 21 \frac{f_{1}^{3} f_{2}^{2} f_{6}^{2}}{f_{3}} \quad(\bmod 27)
$$

Employing (33) into (52), the equation reduces to

$$
\sum_{n=0}^{\infty} \bar{C}_{1,2}^{6}(8 n+5) q^{n} \equiv 21 \frac{f_{2}^{2} f_{4}^{3} f_{6}^{2}}{f_{12}}+18 q \frac{f_{2}^{4} f_{12}^{3}}{f_{4}} \quad(\bmod 27)
$$

which implies

$$
\sum_{n=0}^{\infty} \bar{C}_{1,2}^{6}(16 n+5) q^{n} \equiv 21 \frac{f_{1}^{2} f_{2}^{3} f_{3}^{2}}{f_{6}} \quad(\bmod 27) .
$$

In view of congruences (50) and (53), we see that

$$
\bar{C}_{1,2}^{6}(16 n+5) \equiv 7 \cdot \bar{C}_{1,2}^{6}(4 n+1) \quad(\bmod 27) .
$$

Using the above relation and by induction on $\alpha$, we arrive at (6).

\section{Proof of Theorem 2}

Setting $i=1, j=5$ and $\delta=12$ in (5), we find that

$$
\sum_{n=0}^{\infty} \bar{C}_{1,5}^{12}(n) q^{n}=\frac{f\left(q, q^{11}\right) f\left(q^{5}, q^{7}\right)}{(q ; q)_{\infty}^{2}}=\frac{f_{2}^{2} f_{3} f_{12}^{3}}{f_{1}^{3} f_{4} f_{6}^{2}} .
$$

By the binomial theorem, it is easy to see that for positive integers $k$ and $m$,

$$
\begin{aligned}
f_{2 k}^{m} & \equiv f_{k}^{2 m} \quad(\bmod 2), \\
f_{2 k}^{2 m} & \equiv f_{k}^{4 m} \quad(\bmod 4) .
\end{aligned}
$$


Invoking (56) in (54), we see that

$$
\sum_{n=0}^{\infty} \bar{C}_{1,5}^{12}(n) q^{n} \equiv \frac{f_{1} f_{3} f_{12}^{3}}{f_{4} f_{6}^{2}} \quad(\bmod 4) .
$$

Substituting (39) into (57), we obtain

$$
\sum_{n=0}^{\infty} \bar{C}_{1,5}^{12}(n) q^{n} \equiv \frac{f_{3} f_{9} f_{18}}{f_{6}}-q \frac{f_{3}^{2} f_{18}^{4}}{f_{6}^{2} f_{9}^{2}}-q^{2} \frac{f_{3} f_{9} f_{36}^{3}}{f_{12} f_{18}^{2}} \quad(\bmod 4) .
$$

Extracting the terms involving $q^{3 n}, q^{3 n+1}$ and $q^{3 n+2}$ from the above equation, we obtain respectively (7), (8) and (9).

In view of congruences (9) and (57), we deduce that

$$
\bar{C}_{1,5}^{12}(3 n+2) \equiv 3 \cdot \bar{C}_{1,5}^{12}(n) \quad(\bmod 4) .
$$

Using the above relation and by induction on $\alpha$, we arrive at (10).

\section{Proof of Theorem 3}

Substituting (35) in (7), we find that

$$
\sum_{n=0}^{\infty} \bar{C}_{1,5}^{12}(3 n) q^{n} \equiv \frac{f_{8}^{2} f_{12}^{4}}{f_{4}^{2} f_{24}^{2}}-q \frac{f_{4}^{4} f_{6}^{2} f_{24}^{2}}{f_{2}^{2} f_{8}^{2} f_{12}^{2}} \quad(\bmod 4) .
$$

Collecting the terms involving $q^{2 n}$ from (58) and replacing $q^{2}$ by $q$, we get

$$
\sum_{n=0}^{\infty} \bar{C}_{1,5}^{12}(6 n) q^{n} \equiv \frac{f_{4}^{2} f_{6}^{4}}{f_{2}^{2} f_{12}^{2}} \quad(\bmod 4)
$$

Using (56) in (59), we have

$$
\sum_{n=0}^{\infty} \bar{C}_{1,5}^{12}(6 n) q^{n} \equiv f_{2}^{2} \quad(\bmod 4) .
$$

Extracting the terms involving $q^{2 n+1}$ from (60), we obtain (11).

Extracting the terms involving $q^{2 n+1}$ from (58), dividing by $q$ and replacing $q^{2}$ by $q$, we arrive at

$$
\sum_{n=0}^{\infty} \bar{C}_{1,5}^{12}(6 n+3) q^{n} \equiv 3 \frac{f_{2}^{4} f_{3}^{2} f_{12}^{2}}{f_{1}^{2} f_{4}^{2} f_{6}^{2}} \quad(\bmod 4) .
$$

Invoking (56) in (61), we get

$$
\sum_{n=0}^{\infty} \bar{C}_{1,5}^{12}(6 n+3) q^{n} \equiv 3 \frac{f_{3}^{2} f_{6}^{2}}{f_{1}^{2}} \quad(\bmod 4) .
$$

Employing (36) into (62), we have

$$
\sum_{n=0}^{\infty} \bar{C}_{1,5}^{12}(6 n+3) q^{n} \equiv 3 \frac{f_{4}^{4} f_{6}^{3} f_{12}^{2}}{f_{2}^{5} f_{8} f_{24}}+2 q \frac{f_{4} f_{6}^{4} f_{8} f_{24}}{f_{2}^{4} f_{12}} \quad(\bmod 4) .
$$


Collecting the even term of the above equation, we find that

$$
\sum_{n=0}^{\infty} \bar{C}_{1,5}^{12}(12 n+3) q^{n} \equiv 3 \frac{f_{2}^{4} f_{3}^{3} f_{6}^{2}}{f_{1}^{5} f_{4} f_{12}} \quad(\bmod 4) .
$$

Using (56) in (63), we see that

$$
\sum_{n=0}^{\infty} \bar{C}_{1,5}^{12}(12 n+3) q^{n} \equiv 3 \frac{f_{2}^{2} f_{3}^{3} f_{6}^{2}}{f_{1} f_{4} f_{12}} \quad(\bmod 4) .
$$

Substituting (30) into (64), we obtain

$$
\sum_{n=0}^{\infty} \bar{C}_{1,5}^{12}(12 n+3) q^{n} \equiv 3 \frac{f_{4}^{2} f_{6}^{4}}{f_{12}^{2}}+3 q \frac{f_{2}^{2} f_{6}^{2} f_{12}^{2}}{f_{4}^{2}} \quad(\bmod 4)
$$

which implies that

$$
\sum_{n=0}^{\infty} \bar{C}_{1,5}^{12}(24 n+3) q^{n} \equiv 3 \frac{f_{2}^{2} f_{3}^{4}}{f_{6}^{2}} \quad(\bmod 4)
$$

Invoking (56) in (66), we have

$$
\sum_{n=0}^{\infty} \bar{C}_{1,5}^{12}(24 n+3) q^{n} \equiv 3 f_{2}^{2} \quad(\bmod 4) .
$$

Extracting the terms involving $q^{2 n+1}$ from the above equation, we get (12).

Extracting the terms involving $q^{2 n+1}$ from (65), dividing by $q$ and replacing $q^{2}$ by $q$, we obtain

$$
\sum_{n=0}^{\infty} \bar{C}_{1,5}^{12}(24 n+15) q^{n} \equiv 3 \frac{f_{1}^{2} f_{3}^{2} f_{6}^{2}}{f_{2}^{2}} \quad(\bmod 4) .
$$

Using (56) in (68), we see that

$$
\sum_{n=0}^{\infty} \bar{C}_{1,5}^{12}(24 n+15) q^{n} \equiv 3 \frac{f_{3}^{2} f_{6}^{2}}{f_{1}^{2}} \quad(\bmod 4) .
$$

Employing (36) into (69), we have

$$
\sum_{n=0}^{\infty} \bar{C}_{1,5}^{12}(24 n+15) q^{n} \equiv 3 \frac{f_{4}^{4} f_{6}^{3} f_{12}^{2}}{f_{2}^{5} f_{8} f_{24}}+2 q \frac{f_{4} f_{6}^{4} f_{8} f_{24}}{f_{2}^{4} f_{12}} \quad(\bmod 4)
$$

which implies,

$$
\sum_{n=0}^{\infty} \bar{C}_{1,5}^{12}(48 n+15) q^{n} \equiv 3 \frac{f_{2}^{4} f_{3}^{3} f_{6}^{2}}{f_{1}^{5} f_{4} f_{12}} \quad(\bmod 4) .
$$

Invoking (56) in (71), we get

$$
\sum_{n=0}^{\infty} \bar{C}_{1,5}^{12}(48 n+15) q^{n} \equiv 3 \frac{f_{2}^{2} f_{3}^{3} f_{6}^{2}}{f_{1} f_{4} f_{12}} \quad(\bmod 4) .
$$

In view of congruences (64) and (72), we obtain

$$
\bar{C}_{1,5}^{12}(48 n+15) \equiv \bar{C}_{1,5}^{12}(12 n+3) \quad(\bmod 4) .
$$

Using the above relation and by induction on $\alpha$, we arrive at

$$
\bar{C}_{1,5}^{12}\left(3 \cdot 4^{\alpha+2} n+4 \cdot 4^{\alpha+2}-1\right) \equiv \bar{C}_{1,5}^{12}(12 n+3) \quad(\bmod 4) .
$$


Using the congruence (12) in (73), we obtain (14).

Extracting the terms involving $q^{2 n+1}$ from (70), dividing by $q$ and replacing $q^{2}$ by $q$, we have

$$
\sum_{n=0}^{\infty} \bar{C}_{1,5}^{12}(48 n+39) q^{n} \equiv 2 \frac{f_{2} f_{3}^{4} f_{4} f_{12}}{f_{1}^{4} f_{6}} \quad(\bmod 4) .
$$

Invoking (55) in (74), we get

$$
\sum_{n=0}^{\infty} \bar{C}_{1,5}^{12}(48 n+39) q^{n} \equiv 2 f_{2} f_{6} f_{12} \quad(\bmod 4) .
$$

Congruence (13) follows by extracting the terms involving $q^{2 n+1}$ from the above equation.

\section{Proof of Theorem 4}

Employing (37) into (8), we have

$$
\sum_{n=0}^{\infty} \bar{C}_{1,5}^{12}(3 n+1) q^{n} \equiv 3 \frac{f_{4}^{2} f_{12}^{4}}{f_{2} f_{6} f_{8} f_{24}}+2 q \frac{f_{6}^{3} f_{8} f_{12} f_{24}}{f_{4}} \quad(\bmod 4) .
$$

Extracting the terms involving $q^{2 n}$ from (76) and replacing $q^{2}$ by $q$, we obtain

$$
\sum_{n=0}^{\infty} \bar{C}_{1,5}^{12}(6 n+1) q^{n} \equiv 3 \frac{f_{2}^{2} f_{6}^{4}}{f_{1} f_{3} f_{4} f_{12}} \quad(\bmod 4) .
$$

Substituting (34) into (77), we arrive at

$$
\sum_{n=0}^{\infty} \bar{C}_{1,5}^{12}(6 n+1) q^{n} \equiv 3 \frac{f_{8}^{2} f_{12}^{4}}{f_{4}^{2} f_{24}^{2}}+3 q \frac{f_{4}^{4} f_{6}^{2} f_{24}^{2}}{f_{2}^{2} f_{8}^{2} f_{12}^{2}} \quad(\bmod 4) .
$$

Extracting the terms involving $q^{2 n}$ from (78) and replacing $q^{2}$ by $q$, we deduce that

$$
\sum_{n=0}^{\infty} \bar{C}_{1,5}^{12}(12 n+1) q^{n} \equiv 3 \frac{f_{4}^{2} f_{6}^{4}}{f_{2}^{2} f_{12}^{2}} \quad(\bmod 4) \text {. }
$$

Invoking (56) in (79), we get

$$
\sum_{n=0}^{\infty} \bar{C}_{1,5}^{12}(12 n+1) q^{n} \equiv 3 f_{2}^{2} \quad(\bmod 4) .
$$

Extracting the terms involving $q^{2 n+1}$ from (80), we obtain (15) and combining (67) and (80), we get (16).

From the (78), which implies that

$$
\sum_{n=0}^{\infty} \bar{C}_{1,5}^{12}(12 n+7) q^{n} \equiv 3 \frac{f_{2}^{4} f_{3}^{2} f_{12}^{2}}{f_{1}^{2} f_{4}^{2} f_{6}^{2}} \quad(\bmod 4) .
$$

Using (56) in (81), we have

$$
\sum_{n=0}^{\infty} \bar{C}_{1,5}^{12}(12 n+7) q^{n} \equiv 3 \frac{f_{3}^{2} f_{6}^{2}}{f_{1}^{2}} \quad(\bmod 4) .
$$

Combining (69) and (82), we arrive at (17). 


\section{Proof of Theorem 5}

From (60), we have

$$
\sum_{n=0}^{\infty} \bar{C}_{1,5}^{12}(12 n) q^{n} \equiv f_{1}^{2} \quad(\bmod 4) .
$$

Employing (29) in the above equation and then extracting the terms containing $q^{5 n+2}$, dividing by $q^{2}$ and replacing $q^{5}$ by $q$, we get

$$
\sum_{n=0}^{\infty} \bar{C}_{1,5}^{12}(60 n+24) q^{n} \equiv f_{5}^{2} \quad(\bmod 4),
$$

which yields

$$
\sum_{n=0}^{\infty} \bar{C}_{1,5}^{12}(300 n+24) q^{n} \equiv f_{1}^{2} \equiv \sum_{n=0}^{\infty} \bar{C}_{1,5}^{12}(12 n) q^{n} \quad(\bmod 4) .
$$

By induction on $\alpha$, we obtain (18).

The congruence (19) follows by extracting the terms involving $q^{5 n+i}$ for $i=1,2,3,4$ from both sides of (83).

\section{Proof of Theorem 6}

Extracting the terms involving $q^{2 n}$ from (75) and replacing $q^{2}$ by $q$ we have

$$
\sum_{n=0}^{\infty} \bar{C}_{1,5}^{12}(96 n+39) q^{n} \equiv 2 f_{1} f_{3} f_{6} \quad(\bmod 4) .
$$

Substituting (35) into (85), we arrive at

$$
\sum_{n=0}^{\infty} \bar{C}_{1,5}^{12}(96 n+39) q^{n} \equiv 2 \frac{f_{2} f_{8}^{2} f_{12}^{4}}{f_{4}^{2} f_{24}^{2}}-2 q \frac{f_{4}^{4} f_{6}^{2} f_{24}^{2}}{f_{2} f_{8}^{2} f_{12}^{2}} \quad(\bmod 4) .
$$

Extracting the even terms in the above equation, we obtain

$$
\sum_{n=0}^{\infty} \bar{C}_{1,5}^{12}(192 n+39) q^{n} \equiv 2 \frac{f_{1} f_{4}^{2} f_{6}^{4}}{f_{2}^{2} f_{12}^{2}} \quad(\bmod 4) .
$$

Using (55) in (86), we see that

$$
\sum_{n=0}^{\infty} \bar{C}_{1,5}^{12}(192 n+39) q^{n} \equiv 2 f_{1} f_{4} \quad(\bmod 4) \text {. }
$$

Define

$$
\sum_{n=0}^{\infty} f(n) q^{n}=f_{1} f_{4}
$$

Combining (87) and (88), we find that

$$
\sum_{n=0}^{\infty} \bar{C}_{1,5}^{12}(192 n+39) q^{n} \equiv 2 \sum_{n=0}^{\infty} f(n) q^{n} \quad(\bmod 4) .
$$


For a prime, $p \geq 5$ or $\frac{-(p-1)}{2} \leq k, m \leq \frac{p-1}{2}$, consider

$$
\frac{3 k^{2}+k}{2}+4 \cdot \frac{3 m^{2}+m}{2} \equiv \frac{5 p^{2}-5}{24}(\bmod p),
$$

therefore,

$$
(6 k+1)^{2}+4 \cdot(6 m+1)^{2} \equiv 0 \quad(\bmod p),
$$

Since $\left(\frac{-4}{p}\right)=-1$ the congruence relation (90) holds if and only if both $k=m=\frac{ \pm p-1}{6}$. Therefore, if we substitute Lemma 9 into (88) and then extract the terms in which the powers of $q$ are congruent to $\frac{5 p^{2}-5}{24}$ modulo $p$ and then divide by $q^{\frac{5 p^{2}-5}{24}}$, we find that

$$
\sum_{n=0}^{\infty} f\left(p n+\frac{5 p^{2}-5}{24}\right) q^{p n}=f_{p^{2}} f_{4 p^{2}},
$$

which implies that

$$
\sum_{n=0}^{\infty} f\left(p^{2} n+\frac{5 p^{2}-5}{24}\right) q^{n}=f_{1} f_{4}
$$

and for $n \geq 0$,

$$
f\left(p^{2} n+p i+\frac{5 p^{2}-5}{24}\right)=0,
$$

where $i$ is an integer and $1 \leq i \leq p-1$. By induction, we see that for $n \geq 0$ and $\alpha \geq 0$,

$$
f\left(p^{2 \alpha} n+\frac{5 p^{2 \alpha}-5}{24}\right)=f(n) .
$$

Replacing $n$ by $p^{2 \alpha} n+\frac{5 p^{2 \alpha}-5}{24}$ in (89), we arrive at (20).

\section{Proof of Theorem 7}

Replacing $n$ by $p^{2} n+p i+\frac{5 p^{2}-5}{24}$ in (92) and using (91), we find that for $n \geq 0$ and $\alpha \geq 0$,

$$
f\left(p^{2 \alpha+2} n+p^{2 \alpha+1} i+\frac{5 p^{\alpha+2}-5}{24}\right)=0 .
$$

Comparing coefficients of $q^{n}$ from both sides of (89), we see that for $n \geq 0$,

$$
\bar{C}_{1,5}^{12}(192 n+39) \equiv 2 f(n) \quad(\bmod 4) .
$$

The required result follows from (93) and (94).

\section{Proof of Theorem 8}

Setting $i=3, j=3$ and $\delta=9$ in (5), we have

$$
\sum_{n=0}^{\infty} \bar{C}_{3,3}^{9}(n) q^{n}=\frac{f\left(q^{3}, q^{6}\right) f\left(q^{3}, q^{6}\right)}{(q ; q)_{\infty}^{2}} .
$$


After $q$-product manipulation, we see that

$$
\sum_{n=0}^{\infty} \bar{C}_{3,3}^{9}(n) q^{n}=\frac{f_{6}^{2} f_{9}^{4}}{f_{1}^{2} f_{3}^{2} f_{18}^{2}} .
$$

Invoking (56) in (95), we have

$$
\sum_{n=0}^{\infty} \bar{C}_{3,3}^{9}(n) q^{n} \equiv \frac{f_{3}^{2}}{f_{1}^{2}} \quad(\bmod 4)
$$

Employing (36) into (96), we obtain

$$
\sum_{n=0}^{\infty} \bar{C}_{3,3}^{9}(n) q^{n} \equiv \frac{f_{4}^{4} f_{6} f_{12}^{2}}{f_{2}^{5} f_{8} f_{24}}+2 q \frac{f_{4} f_{6}^{2} f_{8} f_{24}}{f_{2}^{4} f_{12}} \quad(\bmod 4)
$$

Extracting the odd terms of the above equation, we find that

$$
\sum_{n=0}^{\infty} \bar{C}_{3,3}^{9}(2 n+1) q^{n} \equiv 2 \frac{f_{2} f_{3}^{2} f_{4} f_{12}}{f_{1}^{4} f_{6}} \quad(\bmod 4) .
$$

Using (55) in (97), we get

$$
\sum_{n=0}^{\infty} \bar{C}_{3,3}^{9}(2 n+1) q^{n} \equiv 2 f_{2} f_{12} \quad(\bmod 4)
$$

which implies,

$$
\bar{C}_{3,3}^{9}(4 n+3) \equiv 0 \quad(\bmod 4) .
$$

Extracting the even terms from (98), we have

$$
\sum_{n=0}^{\infty} \bar{C}_{3,3}^{9}(4 n+1) q^{n} \equiv 2 f_{1} f_{6} \quad(\bmod 4)
$$

Define

$$
\sum_{n=0}^{\infty} g(n) q^{n}=f_{1} f_{6}
$$

Combining (99) and (100), we find that

$$
\sum_{n=0}^{\infty} \bar{C}_{3,3}^{9}(4 n+1) q^{n} \equiv 2 \sum_{n=0}^{\infty} g(n) q^{n} \quad(\bmod 4) .
$$

For a prime, $p \geq 5$ or $\frac{-(p-1)}{2} \leq k, m \leq \frac{p-1}{2}$, consider

$$
\frac{3 k^{2}+k}{2}+6 \cdot \frac{3 m^{2}+m}{2} \equiv \frac{7 p^{2}-7}{24}(\bmod p),
$$

therefore,

$$
(6 k+1)^{2}+6 \cdot(6 m+1)^{2} \equiv 0 \quad(\bmod p),
$$

Since $\left(\frac{-6}{p}\right)=-1$ the congruence relation (102) holds if and only if both $k=m=\frac{ \pm p-1}{6}$. Therefore, if we substitute Lemma 9 into (100) and then extract the terms in which the powers of $q$ are congruent to $\frac{7 p^{2}-7}{24}$ modulo $p$ and then divide by $q^{\frac{7 p^{2}-7}{24}}$, we find that

$$
\sum_{n=0}^{\infty} g\left(p n+\frac{7 p^{2}-7}{24}\right) q^{p n}=f_{p^{2}} f_{6 p^{2}},
$$


which implies that

$$
\sum_{n=0}^{\infty} g\left(p^{2} n+\frac{7 p^{2}-7}{24}\right) q^{n}=f_{1} f_{6}
$$

and for $n \geq 0$,

$$
g\left(p^{2} n+p i+\frac{7 p^{2}-7}{24}\right)=0,
$$

where $i$ is an integer and $1 \leq i \leq p-1$. By induction, we see that for $n \geq 0$ and $\alpha \geq 0$,

$$
g\left(p^{2 \alpha} n+\frac{7 p^{2 \alpha}-7}{24}\right)=g(n) \text {. }
$$

Replacing $n$ by $p^{2 \alpha} n+\frac{7 p^{2 \alpha}-7}{24}$ in (101), we arrive at (21).

\section{Proof of Theorem 9}

Replacing $n$ by $p^{2} n+p i+\frac{7 p^{2}-7}{24}$ in (104) and using (103), we find that for $n \geq 0$ and $\alpha \geq 0$,

$$
g\left(p^{2 \alpha+2} n+p^{2 \alpha+1} i+\frac{7 p^{\alpha+2}-7}{24}\right)=0 .
$$

Comparing coefficients of $q^{n}$ from both sides of (101), we see that for $n \geq 0$,

$$
\bar{C}_{3,3}^{9}(4 n+1) \equiv 2 g(n) \quad(\bmod 4) .
$$

The required result follows from (105) and (106).

\section{Proof of Theorem 10}

Putting $i=5, j=5$ and $\delta=15$ in (5), we find that

$$
\sum_{n=0}^{\infty} \bar{C}_{5,5}^{15}(n) q^{n}=\frac{f\left(q^{5}, q^{10}\right)^{2}}{(q ; q)_{\infty}^{2}}
$$

After $q$-product manipulation, we see that

$$
\sum_{n=0}^{\infty} \bar{C}_{5,5}^{15}(n) q^{n}=\frac{f_{10}^{2} f_{15}^{4}}{f_{1}^{2} f_{5}^{2} f_{30}^{2}}
$$

Invoking (56) in (107), we have

$$
\sum_{n=0}^{\infty} \bar{C}_{5,5}^{15}(n) q^{n} \equiv \frac{f_{5}^{2}}{f_{1}^{2}} \quad(\bmod 4) .
$$

Substituting (42) into (108), we get

$$
\sum_{n=0}^{\infty} \bar{C}_{5,5}^{15}(n) q^{n} \equiv\left(\frac{f_{8} f_{20}^{2}}{f_{2}^{2} f_{40}}+q \frac{f_{4}^{3} f_{10} f_{40}}{f_{2}^{3} f_{8} f_{20}}\right)^{2} \quad(\bmod 4),
$$


which implies that

$$
\sum_{n=0}^{\infty} \bar{C}_{5,5}^{15}(2 n+1) q^{n} \equiv 2 f_{1} f_{5}^{3} \quad(\bmod 4) .
$$

Employing (43) into (109), we obtain

$$
\sum_{n=0}^{\infty} \bar{C}_{5,5}^{15}(2 n+1) q^{n} \equiv 2 f_{2}^{3} f_{10}+2 q \frac{f_{2}^{2} f_{10}^{2} f_{20}}{f_{4}} \quad(\bmod 4) .
$$

Extracting the terms involving $q^{2 n}$ from (110) and replacing $q^{2}$ by $q$, we arrive at

$$
\sum_{n=0}^{\infty} \bar{C}_{5,5}^{15}(4 n+1) q^{n} \equiv 2 f_{1}^{3} f_{5} \quad(\bmod 4)
$$

Substituting (44) into (111), the equation reduces to

$$
\sum_{n=0}^{\infty} \bar{C}_{5,5}^{15}(4 n+1) q^{n} \equiv 2 \frac{f_{2}^{2} f_{4} f_{10}^{2}}{f_{20}}+2 q f_{2} f_{10}^{3} \quad(\bmod 4),
$$

which implies that

$$
\sum_{n=0}^{\infty} \bar{C}_{5,5}^{15}(8 n+5) q^{n} \equiv 2 f_{1} f_{5}^{3} \quad(\bmod 4) .
$$

Combining (109) and (113), we get

$$
\bar{C}_{5,5}^{15}(8 n+5) \equiv \bar{C}_{5,5}^{15}(2 n+1) \quad(\bmod 4) .
$$

Using the above relation and by induction on $\alpha$, we arrive at (26).

Extracting the terms involving $q^{2 n+1}$ from (110), dividing by $q$ and replacing $q^{2}$ by $q$, we obtain

$$
\sum_{n=0}^{\infty} \bar{C}_{5,5}^{15}(4 n+3) q^{n} \equiv 2 \frac{f_{1}^{2} f_{5}^{2} f_{10}}{f_{2}} \quad(\bmod 4) .
$$

Using (55), one can reduce the above equation to

$$
\sum_{n=0}^{\infty} \bar{C}_{5,5}^{15}(4 n+3) q^{n} \equiv 2 f_{5}^{4} \quad(\bmod 4)
$$

Collecting the terms involving $q^{5 n+i}$ on both sides of (114), we find that

$$
\bar{C}_{5,5}^{15}(4(5 n+i)+3) q^{n} \equiv 0 \quad(\bmod 4), \quad i=1,2,3,4 .
$$

Extracting the terms involving $q^{5 n}$ from (114), we obtain

$$
\sum_{n=0}^{\infty} \bar{C}_{5,5}^{15}(20 n+3) q^{n} \equiv 2 f_{1}^{4} \quad(\bmod 4) \text {. }
$$

Employing (29) into (116) and extracting the terms involving $q^{5 n+4}$ in the resultant equation, we have

$$
\sum_{n=0}^{\infty} \bar{C}_{5,5}^{15}(100 n+83) q^{n} \equiv 2 f_{5}^{4} \quad(\bmod 4) .
$$

In the view of congruences (114) and (117), we obtain

$$
\bar{C}_{5,5}^{15}(100 n+83) q^{n} \equiv \bar{C}_{5,5}^{15}(4 n+3) \quad(\bmod 4) .
$$


Using the above relation and by induction on $\alpha$, we have

$$
\bar{C}_{5,5}^{15}\left(4 \cdot 5^{5 \alpha+5} n+\frac{2 \cdot 5^{5 \alpha+6}-1}{3}\right) \equiv \bar{C}_{5,5}^{15}(4 n+3) \quad(\bmod 4) .
$$

Using congruence (115) in the above equation, we get (27).

Extracting the even terms of (112), we obtain

$$
\sum_{n=0}^{\infty} \bar{C}_{5,5}^{15}(8 n+1) q^{n} \equiv 2 \frac{f_{1}^{2} f_{2} f_{5}^{2}}{f_{10}} \quad(\bmod 4) .
$$

Invoking (55) in (118), we deduce

$$
\sum_{n=0}^{\infty} \bar{C}_{5,5}^{15}(8 n+1) q^{n} \equiv 2 f_{2}^{2} \quad(\bmod 4) .
$$

Congruence (23) follows by extracting the terms involving $q^{2 n+1}$ from the above equation.

Using (55) in (119) implies

$$
\sum_{n=0}^{\infty} \bar{C}_{5,5}^{15}(8 n+1) q^{n} \equiv 2 f_{1}^{4} \quad(\bmod 4) .
$$

Combining (116) and (120), we obtain (24).

Substituting (29) into (109), we arrive at

$$
\sum_{n=0}^{\infty} \bar{C}_{5,5}^{15}(2 n+1) q^{n} \equiv 2 f_{5}^{3} f_{25}\left(a\left(q^{5}\right)-q-\frac{q^{2}}{a\left(q^{5}\right)}\right) \quad(\bmod 4) .
$$

Extracting the terms involving $q^{5 n+j}$ on both sides of (121), we obtain

$$
\sum_{n=0}^{\infty} \bar{C}_{5,5}^{15}(2(5 n+j)+1) \equiv 0 \quad(\bmod 4), \quad j=3,4 .
$$

Extracting the terms involving $q^{5 n+1}$ from (121), dividing by $q$ and replacing $q^{5}$ by $q$, we obtain

$$
\sum_{n=0}^{\infty} \bar{C}_{5,5}^{15}(10 n+3) q^{n} \equiv 2 f_{1}^{3} f_{5} \quad(\bmod 4) .
$$

From (111) and (123), we obtain (25).

Employing (29) into (123), we arrive at

$$
\begin{aligned}
& \sum_{n=0}^{\infty} \bar{C}_{5,5}^{15}(10 n+3) q^{n} \\
\equiv & f_{5} f_{25}^{3}\left(a^{3}\left(q^{5}\right)-3 a^{2}\left(q^{5}\right) q+5 q^{3}-\frac{3 q^{5}}{a^{2}\left(q^{5}\right)}-\frac{q^{6}}{a^{3}\left(q^{5}\right)}\right) \quad(\bmod 4),
\end{aligned}
$$

which implies that

$$
\bar{C}_{5,5}^{15}(10(5 n+k)+3) \equiv 0 \quad(\bmod 4), \quad k=2,4 .
$$


Extracting the terms involving $q^{5 n+3}$ from (124), dividing by $q^{3}$ and replacing $q^{5}$ by $q$, we have

$$
\sum_{n=0}^{\infty} \bar{C}_{5,5}^{15}(50 n+33) q^{n} \equiv 2 f_{1} f_{5}^{3} \quad(\bmod 4) .
$$

Combining (109) and (125), we get

$$
\bar{C}_{5,5}^{15}(50 n+33) \equiv \bar{C}_{5,5}^{15}(2 n+1) \quad(\bmod 4) .
$$

Using the above relation and by induction on $\alpha$, we have

$$
\bar{C}_{5,5}^{15}\left(2 \cdot 5^{2 \alpha+4} n+\frac{4 \cdot 5^{2 \alpha+4}-1}{3}\right) \equiv \bar{C}_{5,5}^{15}(2 n+1) \quad(\bmod 4) .
$$

Using (122) in (126), we obtain (28).

Acknowledgements The authors would like to thank the anonymous referee for helpful comments and suggestions and the second author would like to thank UGC for providing National fellowship for higher education (NFHE), ref. no.F1-17.1/2015-16/NFST-2015-17-ST-KAR-1376.

\section{References}

1. Adiga, C., Berndt, B.C., Bhargava, S., Watson, G.N.: Chapter 16 of Ramanujan's Second Notebook: Theta-Function and q-Series. Memoir, vol. 315. Amer. Math. Soc., Providence (1985)

2. Ahmed, Z., Baruah, N.D.: New congruences for Andrews' singular overpartitions. Int. J. Number Theory 11, 2247-2264 (2015)

3. Andrews, G.E.: Singular overpartitions. Int. J. Number Theory 11, 1523-1533 (2015)

4. Baruah, N.D., Ojah, K.K.: Analogues of Ramanujan's partition identities and congruences arising from the theta functions and modular equations. Ramanujan J. 28, 385-407 (2012)

5. Berndt, B.C.: Ramanujan's Notebooks, Part III. Springer-Verlag, New York (1991)

6. Baruah, N.D., Ojah, K.K.: Partitions with designated summands in which all parts are odd. Integers 15, A9 (2015)

7. Chen, S.-C.: Congruences and asymptotics of Andrews' singular overpartitions. J. Number Theory 164, 343-358 (2016)

8. Chen, S.-C., Hirschhorn, M.D., Sellers, J.A.: Arithmetic properties of Andrews' singular overpartitions. Int. J. Number Theory 11, 1463-1476 (2015)

9. Corteel, S., Lovejoy, J.: Overpartitions. Trans. Am. Math. Soc. 356, 1623-1635 (2004)

10. Cui, S.-P., Gu, N.S.S.: Arithmetic properties of $\ell$-regular partitions. Adv. Appl. Math. 51, 507-523 (2013)

11. Hirschhorn, M.D., Garvan, F., Borwein, J.: Cubic analogues of the Jacobian theta function $\theta(z, q)$. Can. J. Math. 45, 673-694 (1993)

12. Hirschhorn, M.D., Sellers, J.A.: Elementary proofs of parity results for 5-regular partitions. Bull. Aust. Math. Soc. 81, 58-63 (2010)

13. Lovejoy, J.: Gordon's theorem for overpartitions. J. Comb. Theory, Ser. A 103, 393-401 (2003)

14. Mahadeva Naika, M.S., Gireesh, D.S.: Congruences for Andrews' singular overpartitions. J. Number Theory 165, 109-130 (2016)

15. Mahadeva Naika, M.S., Hemanthkumar, B.: Arithmetic properties of 5-regular bipartitions. Int. J. Number Theory 13, 937-956 (2017)

16. Mahadeva Naika, M.S., Shivaprasada Nayaka, S.: Andrews' singular overpartitions with odd parts. Funct. Approx. Comment. Math. 56, 195-209 (2017)

17. Mahadeva Naika, M.S., Shivaprasada Nayaka, S.: Andrews' singular overpartitions without multiples of k. Gulf. J. Math. 5, 18-32 (2017)

18. Mahadeva Naika, M.S., Shivashankar, C.: Congruences for Andrews' singular overpartition pairs. Int. J. Number Theory (to appear) 
19. Ramanujan, S.: Collected Papers. Cambridge University press, Cambridge (1927). Reprinted by Chelsea, New York (1962). Reprinted by the American Mathematical Society, Providence, RI(2000)

20. Sumanth Bharadwaj, H.S.: Personal communication (2016)

21. Xia, E.X.W., Yao, O.X.M.: Analogues of Ramanujan's partition identities. Ramanujan J. 31, 373-396 (2013)

22. Yao, O.X.M.: Congruences modulo 16, 32, and 64 for Andrews' singular overpartitions. Ramanujan J. 43, 215-228 (2017) 